PAPER

\title{
Parkinson's disease patients undershoot target size in handwriting and similar tasks
}

\author{
A W A Van Gemmert, C H Adler, G E Stelmach
}

J Neurol Neurosurg Psychiatry 2003;74:1502-1508

\begin{abstract}
Objectives: Previous research suggested that people with Parkinson's disease are able to increase handwriting stroke size up to $1.5 \mathrm{~cm}$ without an increase of stroke duration; whereas age matched individuals in normal health are able to modulate stroke size without changes in stroke duration for sizes up to $2 \mathrm{~cm}$. This study was designed to test this finding by examining whether sizes larger than $1.5 \mathrm{~cm}$ show different relationships with stroke duration for patients with Parkinson's disease as compared with age matched controls.

Methods: The study included 13 subjects with Parkinson's disease and 13 age matched controls. Participants were required to write a cursive "IIIIIII" pattern, or a cursive "Iililili" pattern without the dots, at a comfortable speed and also as fast as possible, in five different sizes $(1.0,1.5,2.0,3.0$, and $5.0 \mathrm{~cm})$. The participants wrote with a ballpoint pen on a digitiser tablet. The target pattern was displayed at its required size on a screen, but disappeared as soon as the pen touched the surface of the digitiser tablet. Online visual monitoring of the hand was prevented by a cover over the digitiser. After each trial, the recorded movement of the tip of the pen was displayed with two lines to indicate whether the size requirement had been met. The writing conditions were presented in random order and consisted of 12 trials for each participant.

Results: The results demonstrated that stroke size and duration produced by the participants with Parkinson's disease were independently modulated up to $1.5 \mathrm{~cm}$; sizes over $1.5 \mathrm{~cm}$ resulted in progressive undershooting by patients with Parkinson's disease (PD). It was also shown that these participants modulated acceleration measures inefficiently as compared with controls.

Conclusions: The findings suggest that individuals with Parkinson's disease writing at speed produce inadequate stroke sizes when these should equal or exceed $1.5 \mathrm{~cm}$.
\end{abstract}

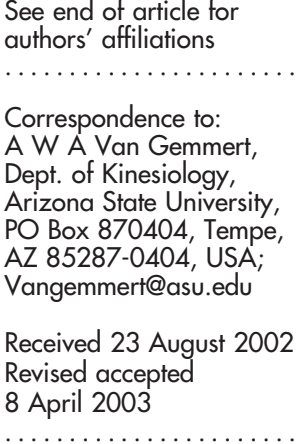

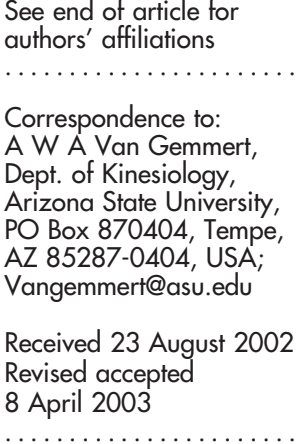

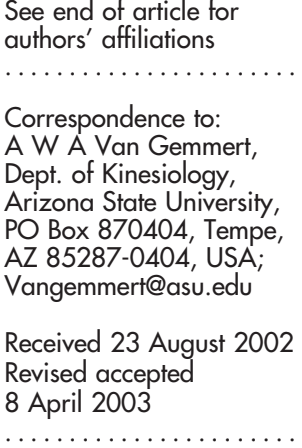

$\mathrm{H}$ andwriting is a communication skill that requires acceleration of the tip of the pen rapidly and accurately over the surface of the paper. An important feature of proficient handwriting is that acceleration needs to be accurately modulated to keep the writing legible. To understand the importance of acceleration measures in graphic tasks, it should noted that acceleration measured at the tip of the pen is a fair approximation of the force needed to propel the tip of the pen over the surface, because frictional forces are relatively low in handwriting. ${ }^{1}$ Furthermore, handwriting movements tend to be executed at the highest comfortable speed at which letter shape variation is still at its minimum. ${ }^{2}$ Because produced forces are low in handwriting, it is assumed that the maximum rate of stroke production for sizes up to $2 \mathrm{~cm}$ is limited by the maximum speed of alternating forces and not by the force needed to generate the particular stroke size. This implies that duration is largely independent of stroke size, according to the isochrony principle. $^{34}$ Therefore, peak acceleration (or peak force, if assumed that frictional forces are negligible) should be proportionally related to stroke size. Furthermore, the time of onset and offset of directional acceleration at the tip of the pen is responsible for letter forms, ${ }^{5}$ which means that acceleration exerted by the wrist and fingers needs to be modulated and coordinated to produce smooth strokes that lead to legible proficiently written pen traces.

Previous research has suggested that control of movement amplitude may be impaired in patients with Parkinson's disease (PD), ${ }^{6-12}$ and several other studies have shown that the control of speed also is impaired in patients with PD. ${ }^{13-16}$ Control of movement amplitude and speed may be the source of two distinctive symptoms often observed in such patients, namely hypometric and bradykinetic movements. Even though these movement features are quite different, they could both originate from a reduced ability to control speed. If patients with PD are less able to increase speed in handwriting, movement amplitude should have a linear relationship to movement duration. Thus, in contrast to the peak acceleration in handwriting of age matched controls, which is governed by the isochrony principle, peak acceleration in parkinsonian handwriting could be unrelated to writing size.

The production of legible handwriting requires accurate coordination between fingers and wrist. Moreover, the coordination between these joints governs the propulsion of the pen tip in the $\mathrm{x}$ and $\mathrm{y}$ directions over the surface. In order to make a sharp reversal, the offsets of the $\mathrm{x}$ and $\mathrm{y}$ directions should coincide, whereas to produce a curved reversal the offsets should not coincide. ${ }^{517}$ Increasing complexity in handwriting therefore could be achieved by continuously changing from curved reversals to sharp reversals, resulting in the inability to use the same coordination plan repeatedly. Several studies of parkinsonian movements showed that patients are more affected by coordination complexity than are age matched controls. ${ }^{18-20}$ Other studies have shown that the ability to switch between subtasks is impaired by PD. ${ }^{21-24}$ Therefore, if it is assumed that an alternating letter patternfor example, $i$ 's (sharp reversals) and $l$ 's (curved reversals) is more complex than a letter pattern of $l$ 's only, patients with PD with should be more affected than age matched controls

Abbreviations: PD, Parkinson's disease; GLM, general linear model 
by the alternating letter pattern, which requires the subject to make alternations between sharp and curved reversals.

In summary, to test the ability of patients with $\mathrm{PD}$ to increase stroke size independently from stroke duration for sizes up to $2 \mathrm{~cm}$, we designed an experiment that used writing patterns which varied in stroke complexity (cursive "llllllll" and "lililili"), speed (comfortable speed and as fast as possible), and required size $(1,1.5,2,3$, and $5 \mathrm{~cm})$. It was predicted that patients with PD would be able to reduce duration to the same extent as healthy age matched controls for the 1, 1.5, and $2 \mathrm{~cm}$ conditions. The 3 and $5 \mathrm{~cm}$ conditions were expected to show an increase in stroke duration as a result of an increase of stroke size for both groups, but stroke durations were expected to increase for these size conditions more for the patients with PD than for the controls, as result of the former's reduced ability to increase acceleration. With respect to the required writing sizes, it was predicted that the patients with PD would be able to produce stroke sizes of 1 and $1.5 \mathrm{~cm}$. When required to produce 2,3 , and $5 \mathrm{~cm}$ stroke sizes, the patients with PD were expected to write in a significantly smaller hand than required, because of their reduced ability to produce a rapid increase of acceleration. These reductions should be magnified for larger sizes. Furthermore, the predicted results were expected to be more pronounced for patients with PD in the complex pattern "lililili" than in the less complex pattern "llllllll", because such patients have a reduced ability to switch between subtasks.

\section{METHOD}

\section{Participants}

Of the 17 persons with PD who volunteered to participate in the experiment, only 13, between the ages of 54 and 82 years (mean age 69.1 years; 4 females and 9 males), were entered in the study; three patients who volunteered were not able to complete all conditions and were therefore not entered. Furthermore, one patient was eliminated because he was only 36 years old. Although this man showed the same general pattern of behaviour in the experimental conditions as older subjects, it was decided to eliminate him to improve age matching. To participate as controls, 13 healthy individuals between the ages of 61 and 78 years (mean age 67.5 years; 8 females and 5 males) were recruited. All patients with PD entered in the study were in the mild stage of the disease (see table 1 for a summary of the characteristics of the patients) and were tested at least 1 hour after medication intake (mean 3.45 hours). All participants were right handed and had normal or corrected to normal vision. The protocol was approved by both the Human Subjects
Institutional Review Board of Arizona State University and the Mayo Clinic, Scottsdale, AZ.

Micrographia was a subjective determination by the patient, whereas bradykinesia, tremor, and rigidity were determined on examination findings.

\section{Task and apparatus}

Participants were required to write either a cursive "llllllll" pattern or a cursive "lililili" pattern without the dots. The first pattern requires repetition of a counterclockwise loop which progresses to the right, whereas the second pattern requires alternation between counterclockwise loops and short strokes with sharp reversals. The alternation of l's and i's is assumed to add control complexity.

The participants were asked to write the patterns at a comfortable speed and also as fast as possible in five different size conditions. The normal writing size is between 0.5 and $1 \mathrm{~cm},{ }^{410}{ }^{11}$ so $1 \mathrm{~cm}$ was chosen as the smallest size condition. The second size condition chosen was $1.5 \mathrm{~cm}$, which has been shown to be within the isochronous size range of patients with PD. ${ }^{10}$ The third size condition chosen was $2.0 \mathrm{~cm}$, which is considered the upper limit of isochronous writing in healthy individuals. ${ }^{410}$ The fourth and fifth size conditions were set at $3.0 \mathrm{~cm}$ and $5.0 \mathrm{~cm}$, respectively. Both the $3.0 \mathrm{~cm}$ and $5.0 \mathrm{~cm}$ conditions are outside the normal isochronous size range, and increases of stroke durations were therefore expected. Whereas $3.0 \mathrm{~cm}$ stroke sizes are performed predominantly with the wrist and fingers, $5.0 \mathrm{~cm}$ stroke sizes are executed by additional use of the elbow joint.

The participants wrote with a normal looking ballpoint pen on a digitiser-tablet (WACOM Intuos $12 \times 18$ ). The digitiser tablet recorded the $\mathrm{x}$ - and $\mathrm{y}$-positions of the tip of the pen with a sampling frequency of $206 \mathrm{~Hz}$ and a spatial resolution of $0.001 \mathrm{~cm}$. The control and collection program for the experiment was written in OASIS. ${ }^{25}$ The digitiser tablet was positioned on a table with a computer display behind it. To prevent online monitoring of the hand, a cover was put over the digitiser tablet. During a trial the pattern was displayed on the screen at its target size. As soon as the pen touched the surface of the digitiser tablet, the pattern disappeared and recording was started. After a minimum of 18 strokes the computer indicated that the trial was finished. If the minimum amount of strokes was not reached within 30 seconds, or if the pattern was not performed correctly, the trial was repeated. After each trial, the recorded movement of the tip of the pen was displayed between two lines to indicate how well the size requirement was met. The experimenter verbally encouraged the participant after each trial to meet the size requirement, and to write as fast as possible if the

\begin{tabular}{|c|c|c|c|c|c|c|c|c|c|c|}
\hline No & A & Sex & $H Y^{*}$ & DS & Mic & Bra & TR & AT & Rig & Med \\
\hline 1 & 76 & Male & 1.5 & Off & No & No & No & Yes & No & $\mathrm{Sn}, \mathrm{Mp}$ \\
\hline 2 & 56 & Female & 2 & No fluctuation & Yes & No & No & Yes & No & $\mathrm{Sn}, \mathrm{Rp}$ \\
\hline 3 & 54 & Male & 2 & Off & No & Yes & Yes & Yes & Yes & $\mathrm{Rp}$ \\
\hline 4 & 69 & Male & 2.5 & Off & Yes & Yes & No & No & Yes & $\mathrm{Sn}, \mathrm{Am}$ \\
\hline 5 & 70 & Female & 3 & No fluctuation & Yes & Yes & Yes & Yes & Yes & $\mathrm{Sn}, \mathrm{Mp}$ \\
\hline 6 & 82 & Female & 1 & On & Yes & Yes & No & No & Yes & $\mathrm{Sn}$ \\
\hline 7 & 79 & Male & 2.5 & No fluctuation & No & Yes & Yes & Yes & Yes & $\mathrm{Sn}, \mathrm{Mp}, \mathrm{Tm}$ \\
\hline 8 & 78 & Male & 3 & Off & Yes & Yes & Yes & Yes & Yes & Sn \\
\hline 9 & 68 & Male & 2.5 & Off & Yes & No & Yes & Yes & Yes & $\mathrm{Sn}, \mathrm{Mp}$ \\
\hline 10 & 62 & Female & 2 & On & Yes & Yes & No & Yes & No & $\mathrm{Sn}, \mathrm{Tm}$ \\
\hline 11 & 61 & Male & 3 & Off & Yes & No & No & No & Yes & $\mathrm{Mp}, \mathrm{Tm}, \mathrm{Cb}$ \\
\hline 12 & 76 & Male & 2 & On & No & Yes & No & No & Yes & Sn,Rp \\
\hline 13 & 67 & Male & 2 & No fluctuation & Yes & Yes & No & No & No & $\mathrm{Am}, \mathrm{Sn}, \mathrm{Rp}, \mathrm{Tm}$ \\
\hline
\end{tabular}


trial was performed in the speed condition. Before the actual experiment started, participants were familiarised with the protocol by extensive practice trials (at least six trials at a comfortable speed and six trials as fast as possible). Thereafter, each condition was started with two practice trials. The conditions were presented in a randomised order and consisted of 12 trials.

\section{Data analysis}

The position data of the handwriting recordings were filtered at $7 \mathrm{~Hz}$ with a dual pass Butterworth 4th order digital filter to eliminate phase shift. After filtering, the patterns were segmented into alternating up and down strokes where the interpolated vertical velocity crossed zero. Segmentation points spaced at less than $0.05 \mathrm{~cm}$ were removed. A pattern verifier checked whether the segmentation and performance of each pattern were correct. This pattern verifier checked for the number of strokes (at least 16 strokes were required) and whether, after any initial downstroke was removed, the first, third, fifth, and seventh stroke pairs were counterclockwise loops with an initial upstroke followed by a downstroke. For the "Illlllll" pattern, stroke sizes larger than $80 \%$ of the maximum measured stroke size in the pattern were required, whereas for the "lililili" pattern, stroke pairs had to alternate between larger than $80 \%$ and smaller than $70 \%$ of the maximum measured stroke size in the pattern. The third, fifth, and seventh stroke pair of both patterns were used for analysis, so the first " $\mathrm{i}$ " was dropped from the analysis to prevent confounding the data with start up effects. The second, fourth, sixth, and eighth stroke pairs were dropped to compare stroke sizes in the "lililili" and "llllllll" patterns, in order to exclude size effects as result of the " $\mathrm{i}$ " which was not targeted in the size conditions.

For every participant, the means of the movement time per stroke (stroke duration), vertical stroke size, mean acceleration, and peak acceleration of the last 10 replications for size and speed conditions were calculated. These means were entered into a General Linear Model (GLM) procedure, according to a completely factorial design with participants nested within groups: 2 groups (controls and patients with PD) $\times 5$ size conditions $(1,1.5,2,3$, and $5 \mathrm{~cm}) \times 2$ speed conditions (comfortable speed and as fast as possible) $\times 2$ patterns ("Illlllll" and "lililili"). Subsequently, if size condition and/or speed and/or pattern significantly interacted with group, a post hoc comparison with Bonferonni correction was performed $(\alpha=0.05)$ to determine which independent cells differed from each other. Additionally, the means of the stroke size were entered into a GLM for each size requirement according to a factorial design with participants nested within groups: 2 groups $\times 2$ speed conditions $\times 2$ patterns, since stroke size was expected to vary with each size condition, and group differences were anticipated to be a function of the size manipulation. If a difference between groups was established with this analysis, then additional two one-sample $t$ tests (one for each group) were performed to determine whether the stroke size deviated significantly from the required size $(\alpha=0.05)$.

\section{RESULTS}

\section{Stroke duration}

The average stroke duration of the patients with PD (384 ms) was longer than the average stroke duration of the controls (309 ms). However, this difference was not significant, $F(1$, $24)=2.40, \mathrm{p}>0.10$. Stroke duration showed significant main effects for pattern $(F(1,24)=4.36, \mathrm{p}<0.05)$, speed $(F(1,24)=32.17, \mathrm{p}<0.001)$, and size $(F(4,96)=23.603$, $\mathrm{p}<0.001$; see table 2$)$. Speed and group, and speed with pattern and group, interacted significantly, $F(1,24)=9.18$, $\mathrm{p}<0.001$, and $F(1,24)=7.26, \mathrm{p}<0.05$, respectively. It was shown that the comfortable stroke duration of the patients with PD (583 ms and $496 \mathrm{~ms}$ for the "Illllll" and "lililili" pattern, respectively) was much longer than the comfortable stroke duration of the controls (366 ms and $347 \mathrm{~ms}$ for the "Illllll" and "lililili" pattern, respectively). The "as fast as possible" condition showed that the patients with PD had slightly shorter stroke durations (220 ms and $236 \mathrm{~ms}$ for the "Illlllll" and "lililili" patterns, respectively) than the controls (264 ms and $260 \mathrm{~ms}$ for the "llllllll" and "lililili" patterns, respectively). However, the size requirement did not affect stroke duration differently in the patients with PD as compared with the controls (see fig 1). No other interactions with group reached significance.

\section{Stroke size}

The patients with PD produced significantly smaller stroke sizes than the controls, $F(1,24)=11.58, \mathrm{p}<0.005$. Stroke size averaged at $19.1 \mathrm{~mm}$ and $25.2 \mathrm{~mm}$ for the patients with $\mathrm{PD}$ and the controls, respectively. Stroke size did not show a significant main effect of speed, $F(1,24)=2.40, \mathrm{p}>0.10$, or pattern $F(1,24)=3.37, \mathrm{p}=0.08$, although a marginal difference was found between the two patterns: see table 2 . Size did show as expected a significant main effect, $F(4,96)$ $=352.30, \mathrm{p}<0.001$ (table 2 ). Group interacted significantly with size, $F(4,96)=6.43, \mathrm{p}<0.001$, pattern and size $F(4,96)$ $=2.72, \mathrm{p}<0.05$, speed and size $F(4,96)=4.52, \mathrm{p}<0.005$, and pattern, speed, and size, $F(4,96)=3.09, \mathrm{p}<0.05$. To reveal the effects of these interactions each size condition was analysed separately.

The analysis for each size condition showed that the patients with PD wrote significantly smaller than the controls in the $1,1.5,2,3$, and $5 \mathrm{~cm}$ conditions (statistics were,

Table 2 Mean values (SEM) of stroke duration, stroke size, mean acceleration, and peak acceleration for main effects of group, speed, size, and pattern

\begin{tabular}{|c|c|c|c|c|c|c|c|c|c|c|c|}
\hline & \multicolumn{2}{|l|}{ Group } & \multicolumn{2}{|l|}{ Speed $^{*}$} & \multicolumn{2}{|l|}{ Pattern* } & \multicolumn{5}{|l|}{ Size$^{*}(\mathrm{cms})$} \\
\hline & Controls & PD & Comf & Fast & |IIIIIIII & lililili & 1.0 & 1.5 & 2.0 & 3.0 & 5.0 \\
\hline $\begin{array}{l}\text { Stroke duration } \\
\text { (ms) }\end{array}$ & 309 (7) & 384 (17) & $448(15)$ & $245(8)$ & $358(15)$ & 335 (12) & $288(18)$ & $306(18)$ & $326(20)$ & $382(23)$ & $429(24)$ \\
\hline Stroke size $(\mathrm{mm})$ & $25.2(0.8)$ & $19.1(0.9)$ & $23.1(0.9)$ & $21.3(0.9)$ & $22.6(0.9)$ & $21.8(0.9)$ & $9.6(0.3)$ & $13.8(0.4)$ & $17.2(0.6)$ & $25.7(0.8)$ & $44.4(1.3)$ \\
\hline $\begin{array}{l}\text { Mean } \\
\text { acceleration } \\
\left(\mathrm{cm} / \mathrm{s}^{2}\right)\end{array}$ & $139(7.2)$ & $79(5.2)$ & $77(3.6)$ & $142(8.0)$ & $113(7.5)$ & $106(5.3)$ & $74(5.6)$ & $94(7.3)$ & $104(9.4)$ & $119(10.5)$ & 155 (14.7) \\
\hline $\begin{array}{l}\text { Peak } \\
\text { acceleration } \\
\left(\mathrm{cm} / \mathrm{s}^{2}\right)\end{array}$ & $239(11.2)$ & $137(8.2)$ & $139(5.6)$ & 237 (12.7) & $193(11.7)$ & $184(8.6)$ & $127(8.8)$ & $161(11.4)$ & 178 (14.4) & 206 (16.5) & $268(23.2)$ \\
\hline
\end{tabular}




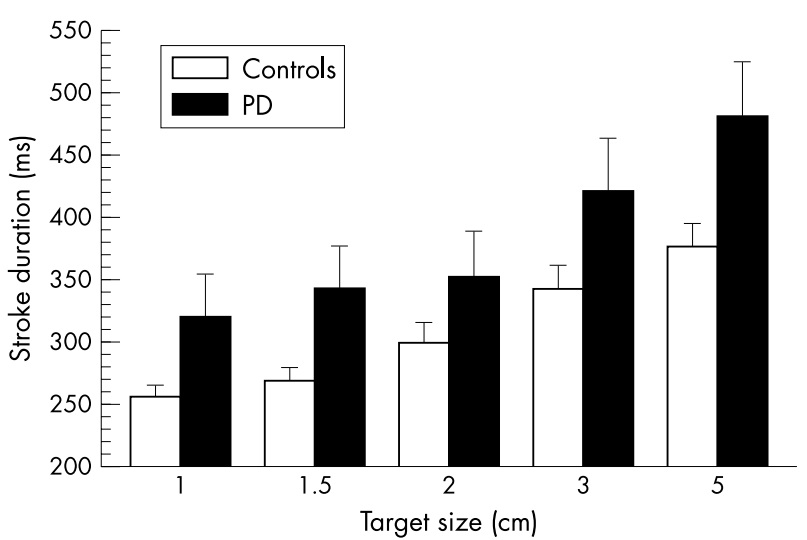

Figure 1 Stroke duration as function of target size and group. PD, patients with Parkinson's disease.

respectively, $F(1,24)=9.09, \mathrm{p}<0.01 ; F(1,24)=4.06$, $\mathrm{p}=0.06 ; F(1,24)=14.68, \mathrm{p}<0.005 ; F(1,24)=13.58$, $\mathrm{p}<0.005 ; F(1,24)=8.65, \mathrm{p}<0.01)$. One-sample t-tests revealed that patients with $\mathrm{PD}$ did not write significantly smaller than required in the 1.0 and $1.5 \mathrm{~cm}$ conditions (fig 2). They did, however, produce significantly smaller stroke sizes than required for the 2,3 , and $5 \mathrm{~cm}$ conditions (fig 2). The controls wrote significantly larger in the $1 \mathrm{~cm}$ condition (which explains why the patients with PD were writing smaller than the controls), but they did not write significantly differently from what was required in the 1.5 , 2,3 , and $5 \mathrm{~cm}$ conditions (see fig 2).

Furthermore, in the 1.0, 1.5, 2.0, 3.0, and $5.0 \mathrm{~cm}$ conditions, group interacted significantly with speed (respectively, $F(1,24)=6.02, \mathrm{p}<0.05 ; F(1,24)=9.37, \mathrm{p}<0.01$; $F(1,24)=4.60, \mathrm{p}<0.05 ; F(1,24), \mathrm{p}<1.0 ; F(1,24)=9.32$, $\mathrm{p}<0.01)$. To investigate whether the sizes differed from the required target size, one-sample t-tests were conducted. These revealed that the controls did not significantly differ from the required size when they used their preferred speed. When writing as fast as possible, the controls did write larger than required in the 1.0 and $2.0 \mathrm{~cm}$ conditions, but not significantly differently from the target size in the 1.5, 3.0, and $5.0 \mathrm{~cm}$ conditions. When writing at a comfortable speed, the patients with PD did write as large as required in the 1.0 and $1.5 \mathrm{~cm}$ conditions, but their writing was significant smaller than required in the $2.0,3.0$, and $5.0 \mathrm{~cm}$ conditions. The "as-fast-as-possible" condition revealed that the patients with PD only kept writing at the required size in the $1.0 \mathrm{~cm}$

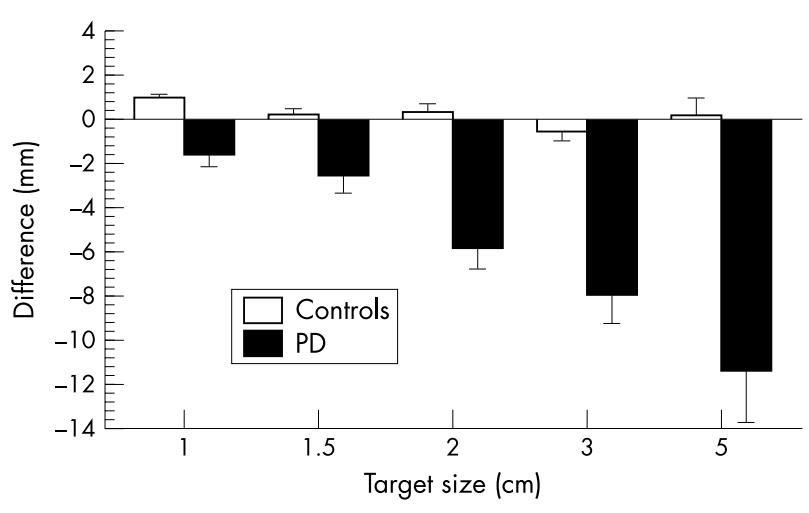

Figure 2 Difference between writing size and target size as function of target size and group. PD, patients with Parkinson's disease.
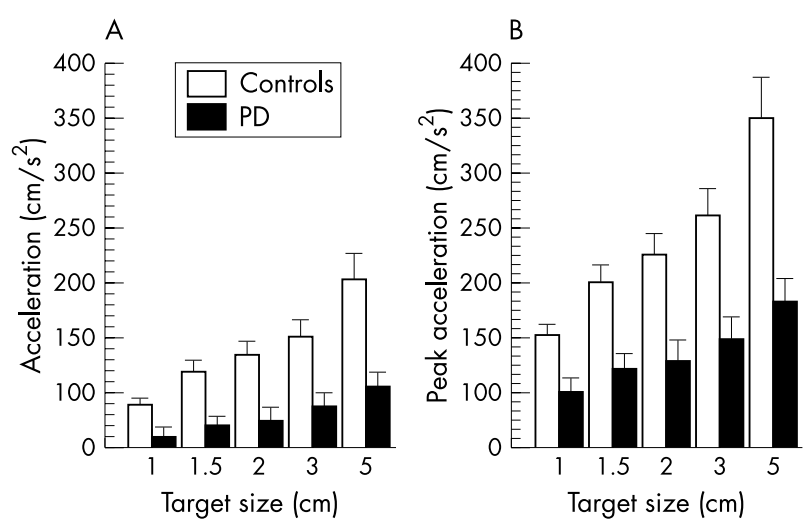

Figure 3 (A) Mean acceleration as function of target size and group. (B) Peak acceleration as function of target size and group. PD, patients with Parkinson's disease.

condition, whereas they increasingly undershot the writing size conditions of $1.5,2.0,3.0$, and $5.0 \mathrm{~cm}$ ).

In the 2.0 and $3.0 \mathrm{~cm}$ conditions, group interacted significantly with speed and pattern, $F(1,24)=4.29$, $\mathrm{p}<0.05 ; F(1,24)=12.28, \mathrm{p}<0.005$, respectively. These interactions showed that whereas the controls kept stroke sizes at approximately 2 and $3 \mathrm{~cm}$ for both speed and pattern conditions, the patients with PD tended to undershoot the required size, and this was more pronounced for the "lililili" pattern. Furthermore, in contrast to the controls, in the "as fast as possible" condition the patients with PD undershot the requested size even more. None of the other size conditions showed higher order interactions with group.

\section{Acceleration}

Mean acceleration of the patients with PD $\left(79.3 \mathrm{~cm} / \mathrm{s}^{2}\right)$ was significantly lower than mean acceleration of the controls $\left(139.3 \mathrm{~cm} / \mathrm{s}^{2}\right), F(1,24)=5.31, \mathrm{p}<0.05$. Size and speed showed significant main effects on mean acceleration, $F(4,96)=20.48, \mathrm{p}<0.001$ and $F(1,24)=15.04, \mathrm{p}<0.005$, respectively (see table 2 ). The two patterns did not show different mean acceleration, $F(1,24)=1.38, \mathrm{p}>0.10$ (see table 2). Mean acceleration showed an interaction with group and size, $F(1,24)=3.49, \mathrm{p}<0.05$. The patients with PD did not scale mean acceleration as much as the controls when larger writing sizes were required. (In the $5.0 \mathrm{~cm}$ condition when compared with the $1.0 \mathrm{~cm}$ condition, the patients with PD increased acceleration by $79 \%$, whereas the controls increased acceleration by $130 \%$; see fig 3). None of the other interactions with group proved to be significant.

\section{Peak acceleration}

The patients with PD reached a significantly smaller peak acceleration $\left(137.0 \mathrm{~cm} / \mathrm{s}^{2}\right)$ than the controls $\left(239.2 \mathrm{~cm} / \mathrm{s}^{2}\right)$, $F(1,24)=6.48, \mathrm{p}<0.05$. As with mean acceleration, both size and speed showed significant main effects on peak acceleration: $F(4,96)=24.11, \mathrm{p}<0.001$ and $F(1,24)=$ 13.73, $\mathrm{p}<.005$, respectively; the two patterns did not differ significantly on this variable, $F(1,24)=1.23, \mathrm{p}>0.10$ (see table 2). Peak acceleration did interact with group and size, $F(4,96)=3.96, \mathrm{p}<0.01$. In addition to the smaller increase in mean acceleration, the increase of peak acceleration also was smaller for the patients with PD (83\%) as compared with the controls (130\%; see fig 3 ) when the target size requirement increased from $1.0 \mathrm{~cm}$ to $5.0 \mathrm{~cm}$. Additional analysis showed that the controls used a more symmetrical time scheme to accelerate and decelerate within a stroke than did the patients with PD. As can be seen in the example shown in fig 4 , the patients with PD showed multiple peaks 

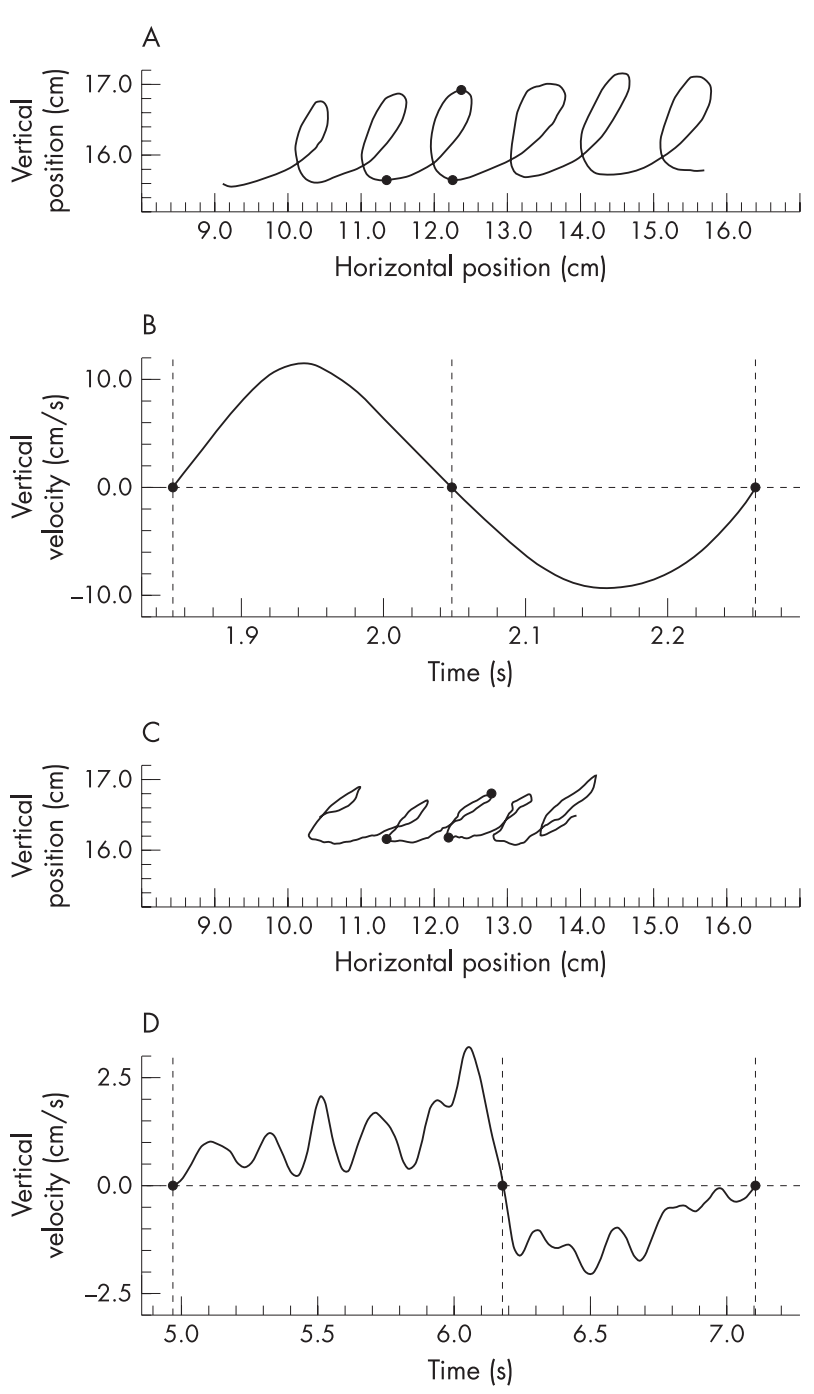

Figure 4 (A) The writing trace of a control elderly individual (the dots indicate the segmentation points for strokes). (B) The velocity signal of the up- and downstroke of the " $" 1$ " in (A) (the dots indicate the segmentation points for the strokes); (C) The writing trace of a patient with Parkinson's disease; (D) The velocity signal of the up- and downstroke of the " $\mu$ " in (C) (note that the scale on the horizontal and vertical axes is different from (B)).

per stroke in the velocity signal (indicating multiple accelerations and decelerations), whereas the controls showed just one peak per stroke (indicating just one acceleration phase and one deceleration phase).

\section{DISCUSSION}

In this study neither the controls nor the participants with PD altered stroke duration if stroke size requirements were set at $1,1.5$, or $2 \mathrm{~cm}$. Furthermore, it was shown that if size requirements were $3 \mathrm{~cm}$ or $5 \mathrm{~cm}$, the patients produced similar increases in stroke durations compared with controls. When writing at a comfortable speed, it was also observed that for sizes to $1.5 \mathrm{~cm}$ controls and participants with PD were equally able to increase stroke size independently of stroke duration. In contrast to the controls, the patients undershot the required size when they were required to produce stroke sizes of $2.0 \mathrm{~cm}$ or larger. In addition, when required to write as fast as possible the patients with PD undershot the required stroke size of $1.5 \mathrm{~cm}$. The controls wrote larger in the "as fast as possible" condition for the
$1.0 \mathrm{~cm}$ to $2.0 \mathrm{~cm}$ target sizes, but matched the required sizes in other size conditions. As expected, an interaction between groups, pattern complexity, and speed requirement was found in the 2.0 and $3.0 \mathrm{~cm}$ stroke size conditions. The patients with PD undershot the "lililili" pattern more than the "llllllll" pattern, and this was accentuated in the "as fast as possible" condition. Finally, when larger stroke sizes were attempted, it was shown that the patients with PD did not increase acceleration and peak acceleration to the same extent as controls.

In a previous study, we suggested that the range within which stroke size can be produced independently of stroke duration (the isochronic size range) for parkinsonian handwriting is not as wide as for controls, ${ }^{10}$ however, participants were not required to write at a certain size, the requirement being to write twice as large. Also, even though real-time visual feedback was provided to the participants, they did not receive feedback about any differences between performed stroke sizes and required stroke sizes to meet the twice as large as normal requirement. Therefore, an alternative explanation for the results of this study could not be refuted. This alternative explanation would suggest that patients with PD suffered from perception action mismatch. The patients would perceive that they had written twice as large, even though they in fact failed to do so. Another difference with the previous study ${ }^{10}$ is that this did not study sizes above the isochronic size range; thus the boundary of the isochronic size range and the duration/size trade off relation above this range were not tested. The present study was designed to fill this gap in knowledge and to determine whether the perception action mismatch hypothesis could explain the findings in our earlier study. Thus, the main difference in experimental design is that the stroke size in the present study was manipulated by requiring the participants to match stroke size to a target stroke size, which enabled us to determine more thoroughly the range in which stroke duration is not significantly affected by increases in stroke size.

It was shown that when writing at a comfortable speed, patients with PD, like the controls, kept stroke durations equal for sizes up to $2.0 \mathrm{~cm}$. However, in contrast to controls, patients with PD undershot the target size of $2.0 \mathrm{~cm}$ and, when required to write "as fast as possible", they even undershot the $1.5 \mathrm{~cm}$ target size. These findings support the view (suggested in our earlier study ${ }^{10}$ ) that the range in which stroke size can be manipulated without significant changes in stroke duration is smaller in parkinsonian handwriting than in the handwriting of controls, and they support the notion that patients with PD tend to trade off stroke size for stroke duration. The latter notion is further supported by the finding that parkinsonian stroke duration was proportionally increased in a similar fashion to that of the controls for target sizes of 3.0 and $5.0 \mathrm{~cm}$, but that these target sizes were increasingly undershot compared with the $2.0 \mathrm{~cm}$ target size.

The target sizes of 2.0 and $3.0 \mathrm{~cm}$ showed a stroke duration for stroke size trade off for the more complex "lililili" pattern in the "as fast as possible" condition. However, the $5.0 \mathrm{~cm}$ condition did not show this trade off. This finding did contrast with the expectation that the $5.0 \mathrm{~cm}$ condition would show an even larger trade off than the 2.0 and $3.0 \mathrm{~cm}$ conditions. A possible explanation for this unexpected result is that $5.0 \mathrm{~cm}$ is a relatively small size for the elbow and shoulder joints, which are expected to be more involved in movements over $3.0 \mathrm{~cm}$ than wrist and finger joints. The elbow and shoulder joints could be resistant for motor load when executing movements with small amplitudes. Thus the extent of undershooting for the $5.0 \mathrm{~cm}$ condition is partly offset by the increased involvement of elbow and shoulder 
joints. This hypothesis is supported by the finding that the trade off was not found for the 1.0 and $1.5 \mathrm{~cm}$ sizes, which are sizes that can be performed comfortably with the finger and wrist joints. However, further corroborated findings for elbow and shoulder joints with relatively large target sizes are necessary to support this notion.

This experiment also showed that increasing target size did not increase mean acceleration and peak acceleration to the same extent for patients with PD as for controls. These findings, together with the findings that (a) stroke durations of patients with PD and of controls were similarly affected by target size; and (b) in contrast to the controls, stroke sizes of patients with PD were smaller than required for target sizes above $1.5 \mathrm{~cm}$, support the notion that inadequate matching to the required target size is related to acceleration inefficiencies. Also, the feature of multiple accelerations in a single stroke supports the view that acceleration inefficiency is related to failure to match the larger target sizes.

In handwriting tasks frictional forces are low, and acceleration is a rough estimation of the forces involved in propelling the pen over the surface. Thus if increases of mean acceleration and peak acceleration due to size increases appear to be reduced, this can be interpreted as a deficiency in the ability to develop force sufficiently, resulting in lower maximum forces and lower rates of force development. The facts that both mean acceleration and peak acceleration were inadequately increased to match the larger stroke sizes, and that, in contrast to controls, patients with PD showed multiple accelerations and decelerations per stroke, support the hypothesis that patients have a reduced ability to modulate forces. The findings are in agreement with several studies, which showed that patients with PD are impaired in modulating muscle activation and/or muscle force..$^{26-29}$

In a wider context, this study shows that even though patients with PD can increase movement amplitude, they are less efficient at modulating the necessary force parameters. The lack of efficiency in force modulation may also play a role in other motor tasks, such as walking. Gait is often characterised by a slow, short stepped, shuffling pattern. ${ }^{30}$ which implies patients have a reduced ability to scale the stride length. ${ }^{31}$ Similarly, Ebersbach and colleagues proposed that the reduced stride length observed in PD is due to a reduction of force gain. ${ }^{32}$ Although walking is quite different in nature from fine motor tasks, such as handwriting, the reduction in stride length is quite similar to the inability to scale stroke sizes. Moreover, these data are in agreement with those studies where hypometria is reported. The fact that patients with PD can increase stroke sizes in handwriting when the size requirements are small, but undershoot larger stroke size requirements, indicates that their limits are not due to a generalised deficit in force control. Furthermore, the observation that patients with PD show multiple peaks in their velocity signal supports the view that they are not suffering from a generalised force deficit. It can be concluded that fine modulation of force is affected rather than increasing the gain of force.

In summary, the findings in this study show that in graphic tasks patients with PD limit stroke duration increases. The consequence is that the stroke amplitude is reduced when targeted stroke size requirements are larger than $1.5 \mathrm{~cm}$. Since handwriting tends to be produced at the highest speed at which the variation of letter shape is kept at a minimum, ${ }^{2}$ the findings support the view that the patients have a reduced isochronic size range. This conclusion is also supported by the finding that when patients with PD wrote as fast as possible, they did produce the required stroke size for the $1.0 \mathrm{~cm}$ target and undershot the $1.5 \mathrm{~cm}$ stroke size. However, age matched controls showed independence between duration and size for targets up to $2 \mathrm{~cm}$. In patients, the control of stroke size and the control of stroke duration are inversely related for stroke sizes of $1.5 \mathrm{~cm}$ and over, resulting in a trade off between size and duration. Furthermore, patients showed that they progressively undershot the required sizes above $1.5 \mathrm{~cm}$, which appears to be related to the inadequate scaling of acceleration and peak acceleration. This supports the suggestion made in our earlier work $^{10}$ that patients with PD are unable sufficiently to modulate force to meet size demands in graphic tasks.

\section{ACKNOWLEDGEMENTS}

This research is supported by a National Institute of Neurological Disorders and Stroke grant, NS 33173, awarded to George E Stelmach.

Portions of the results of this study were presented at the 10th Biennial Conference of the International Graphonomics Society, Nijmegen, The Netherlands, 2001.

\section{Authors' affiliations}

Arend W A Van Gemmert, George E Stelmach, Motor Control Laboratory, Department of Kinesiology, Arizona State University, Tempe, USA

Charles H Adler, Parkinson's Disease and Movement Disorders Center, Mayo Clinic Scottsdale, Scottsdale, USA

\section{REFERENCES}

1 Denier van der Gon JJ, Thuring JP. The guiding of human writing movements. Kybernetik 1965;2:145-8.

2 Wright CE. Evaluating the special role of time in the control of handwriting. Acta Psychol 1993;82:5-52.

3 Viviani $\mathbf{P}, \mathrm{McC}$ Collum $\mathrm{G}$. The relation between linear extent and velocity in drawing movements. Neuroscience 1983;10:211-18.

4 Thomassen AJWM, Teulings HL. Time, size, and shape in handwriting: exploring spatio-temporal relationships at different levels. In: Michon JA, Jackson JB, eds. Time, mind, and behavior. Heidelberg: Springer, 1985:253-63.

5 Schomaker LRB, Thomassen AJWM, Teulings HL. A computational model of cursive handwriting. In: Plamondon R, Suen CY, Simner M, eds. Computer recognition and human production of handwriting. Singapore: World Scientific, Singapore, 1989:153-77.

6 Flash T, Inzelberg R, Schechtman E, et al. Kinematic analysis of upper limb trajectories in Parkinson's disease. Exp Neurol 1992;118:215-26.

7 Margolin DI, Wing AM. Agraphia and micrographia: clinical manifestations of motor programming and performance disorders. Acta Psychol 1983:54:263-83

8 Muller F, Stelmach GE. Prehension movements in Parkinson's disease. In: Stelmach GE, Requin J, eds. Tutorials in motor behavior II. Amsterdam: Elsevier Science, 1992:307-19.

9 Contreras-Vidal JL, Teulings HL, Stelmach GE. Micrographia in Parkinson's disease. Neuroreport 1995:6:2089-92.

10 Van Gemmert AWA, Teulings HL, Contreras-Vidal JL, et al. Parkinson's disease and the control of size and speed in handwriting. Neuropsychologia 1999;37:685-94

11 Van Gemmert AWA, Teulings HL, Stelmach GE. Parkinsonian patients reduce their stroke size with increased processing demands. Brain Cogn 2001;47:504-12.

12 Teulings HL, Contreras-Vidal JL, Stelmach GE, et al. Adaptation of handwriting size under distorted visual feedback in patients with Parkinson's disease and elderly controls and young controls. J Neurol Neurosurg Psychiatry 2002:72:315-24.

13 Nutt JG, Lea ES, Van Houten L, et al. Determinants of tapping speed in normal control subjects and subjects with Parkinson's disease: differing effects of brief and continued practice. Mov Disord 2000;15:843-9.

14 Gordon AM. Task-dependent deficits during object release in Parkinson's disease. Exp Neurol 1998;153:287-98.

15 Tresilian JR, Stelmach GE, Adler CH. Stability of reach-to-grasp movement patterns in Parkinson's disease. Brain 1997; 120:2093-111.

16 Rand MK, Stelmach GE, Bloedel JR. Movement accuracy constraints in Parkinson's disease patients. Neuropsychol 2000;38:203-12.

17 Teulings HL, Stelmach GE. Force amplitude and force duration in parkinsonian handwriting. In: Requin J, Stelmach GE, eds. Tutorials in motor neuroscience. Dordrecht: Kluwer, 1991:149-60.

18 Seidler RD, Alberts JL, Stelmach GE. Multijoint movement control in Parkinson's disease. Exp Brain Res 2001;140:335-44.

19 Alberts JL, Saling M, Adler CH, et al. Disruptions in the reach-to-grasp actions of Parkinson's patients. Exp Brain Res 2000;134:353-62

20 Teulings HL, Contreras-Vidal JL, Stelmach GE, et al. Coordination of fingers, wrist, and arm in parkinsonian handwriting. Exp Neurol 1997; 146:159-70

21 Inzelberg R, Plotnik $M$, Flash T, et al. Mental and motor switching in Parkinson's disease. J Mot Behav 2001;33:377-85.

22 Weiss $\mathbf{P}$, Stelmach GE, Hefter $\mathrm{H}$. Programming of a movement sequence in Parkinson's disease. Brain 1997;120:91-102. 
23 Agostino R, Berardelli A, Formica A, et al. Sequential arm movements in patients with Parkinson's disease, Huntington's disease, and dystonia. Brain 1992; 115: 1481-95.

24 Harrington DL, Haaland KY. Sequencing in Parkinson's disease. Abnormalities in programming and controlling movement. Brain 1991;114:99-115.

25 De Jong WP, Hulstijn W, Kosterman BJM, et al. OASIS soffware and its application in experimental handwriting research. In: Simner ML, Leedham CG, Thomassen AJW, eds. Handwriting and drawing research: basic and applied issues. Amsterdam: IOS Press, 1996:429-40.

26 Hatzitaki V, Hoshizaki TB. Dynamic joint analysis as a method to documen coordination disabilities associated with Parkinson's disease. Clin Biomech 1998; 13:182-9.
27 Pfann KD, Buchman AS, Comella CL, et al. Control of movement distance in Parkinson's disease. Mov Disord 2001; 16:1048-65.

28 Robichaud JA, Pfann KD, Comella CL, et al. Effect of medication on EMG patterns in individuals with Parkinson's disease. Mov Disord. In press.

29 Berardelli A, Rothwell JC, Thompson PD, et al. Pathophysiology of bradykinesia in Parkinson's disease. Brain 2001;124:2131-46.

30 Morris ME, Huxham F, McGinley J, et al. The biomechanics and motor control of gait in Parkinson disease. Clin Biomech 2001;16:459-70.

31 Morris $M$, lansek R, Matyas T, et al. Abnormalities in the stride length-cadence relation in parkinsonian gait. Mov Disord 1998;13:61-9.

32 Ebersbach G, Sojer M, Valldeoriola F, et al. Comparative analysis of gait in Parkinson's disease, cerebellar ataxia and subcortical arteriosclerotic encephalopathy. Brain 1999;122:1349-1355.

\section{$\mathrm{ECHO}$}

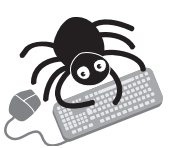

Please visit the Journal of

Neurology, Neurosurgery, and Psychiatry website [www.jnnp. com] for a link to the full text of this article.

\section{CO poisoning seems to spare memory}

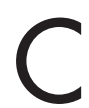

arbon monoxide (CO) poisoning seems not to harm memory in the short term, according to a controlled preliminary study that has used objective psychometric testing for the fist time.

Functional memory and attention and memory one month after intoxication in a highly selected group of 32 patients with no other risk factors for impaired memory were similar to those of matched controls. Some aspects-verbal significance, recall, quality learning (committing to memory), and reaction time-were significantly better. The patients were also significantly better at quality learning, immediate visual memory, and constancy of effort if their initial blood CO concentration was high, though this may be attributed merely to better effort and should be tested in other studies, the authors say.

Patients and controls had a median age of 27.5; 20 were women. Median initial CO concentration was 2.08 (range 1.00-8.58) mmol/l, and 24 had been treated with hyperbaric oxygen. The patients studied were selected from 944 consecutive patients over four years treated for acute CO poisoning by standard normobaric or hyperbaric oxygen therapy. The selection criteria were age 18-60 years, no risk factors for impaired memory, and a minimum CO concentration $\geqslant 1.0 \mathrm{mmol} / \mathrm{l}$ in the first blood sample. They were tested one month after their poisoning for various aspects of memory, attention, and divided attention by psychometric tests.

Subtle neuropsychological effects have been described shortly after acute CO poisoning, but these have been based on subjective testing or without controls for comparison, or both.

A Occupational and Environmental Medicine 2003:60:212-216. 